\title{
Assessment of Knowledge Levels on Malaria among Mothers in Kisumu East Sub-County, Kenya
}

\author{
Walter Ochieng Okande ${ }^{1,2^{*}}$, Gilbert Bor ${ }^{2}$, Fredrick Odoyo ${ }^{2}$ \\ ${ }^{7}$ Moi University, Department of Public Health Services, P. O. Box 3900-30100, Eldoret, Kenya \\ ${ }^{2}$ The Catholic University of Eastern Africa, Department of Post Graduate Studies, Faculty of Arts \& Social Sciences, P. \\ O. Box 908-30100, Eldoret, Kenya
}

\section{*Corresponding Author}

Walter Ochieng Okande

\author{
Article History \\ Received: 20.09 .2020 \\ Accepted: 28.09.2020 \\ Published: 02.10.2020
}

\begin{abstract}
Malaria is a third leading killer in Kenya and number one cause of morbidity and mortality in Kisumu East Sub-County (KESC) among under-fives. Study assessed levels of malaria awareness and knowledge on signs and symptoms among mothers. This was a cross sectional survey with 384 mothers, 2 health providers and 4 chiefs responders. KESC was purposively selected, 4 locations randomly sampled and mothers enrolled into the study by snowball sampling. Data was collected by Semi-structured questionnaires and Interview guides. Analysis was by descriptive and inferential statistics with significance level set at 0.05 . From the results, approximately 383(99\%) mothers knew mosquitoes transmit malaria, 378(98\%) knew mosquitoes infected by malaria parasites cause the disease while 300(78\%) knew mosquitoes breed in stagnant waters or swampy places. Hypothesis findings ( $\mathrm{p}=0.023)$. Moreover, up to $99 \%$ of them knew malaria signs and symptoms. Further, 380(100\%) recognized vomiting, 378(99\%) said fever, $374(98 \%)$ knew appetite loss and 102(27\%) headache as malaria signs in children. Similarly, 378(99\%) knew inability to eat, drink or breast feed, 378(99\%) recognized high body temperature, 268(71\%) convulsion while 254(64\%) mentioned vomiting as severe malaria signs in children. Additionally, 357(93\%) mentioned headache, 339(88\%) said fever, $333(87 \%)$ loss of appetite and 283(74\%) joint pain, backache and body weakness as signs in pregnancy. Hypothesis showed, ( $\mathrm{p}=0.039)$. Based on the results, it is concluded that most mothers knew malaria related information including signs and symptoms despite misconceptions. Therefore, there should be continuous health education through Radio stations, television and us of mobile telephony. There was also significant associations between levels of malaria awareness, signs and symptoms and malaria prevention.
\end{abstract}

Keywords: Malaria, Awareness, Malaria-vulnerable groups, Kenya.

\section{INTRODUCTION}

Worldwide, 3.2 billion people are at risk of malaria with the most vulnerable group being children under 5 and pregnant women in high risk regions [1]. Similarly, every year, 88\% of all malaria cases and 90\% of all global deaths are from Sub-Saharan Africa [1]. In Kenya, over $70 \%$ of the population are at high risk of malaria especially those living in malaria endemic regions [2,3]. Furthermore, malaria is the third leading causes of morbidity and mortality affecting mostly under 5 children and pregnant women [2,3]. In addition, the disease claim over $30 \%$ of all outpatient clinic attendances and up to $19 \%$ of all admissions reported in health institutions in the country in 2018 [4].

Therefore, in order to resolve malaria problem, the Kenya government launched its second National Malaria Strategy meant to reverse malaria cases by $2 / 3$ between the period of 2007 and 2018 [5]. Similarly, this was in line with the UN, SDG 3, WHO, RBM Strategy [6] and the Kenya Vision 2030 [7]. However, despite efforts made, malaria is still the third leading killer in Kenya and number one cause of morbidity and mortality in Kisumu East Sub-County (KESC) among under-fives and in pregnant women [8].

Copyright @ 2020: This is an open-access article distributed under the terms of the Creative Commons Attribution license which permits unrestricted use, distribution, and reproduction in any medium for non commercial use (NonCommercial, or CC-BY-NC) provided the original author and source are credited. 
Most studies conducted previously focused on the knowledge, attitudes and practices regarding malaria [9-12]. For instance, some studies targeted primary care givers (women), under 5 children including pregnant women in highrisk regions $[12,8,11,13]$. Others studies concentrated on gender and malaria $[14,15]$, media exposure and its impact on prevention practices [16] and current strategies and successes of engaging women in vector management at community level [17].

Of all the studies reviewed, none was found on assessment of level of awareness and on recognition of signs and symptoms of malaria among under five children and in pregnant women in the area. Therefore, this study aimed to evaluate the level of awareness and knowledge on signs and symptoms of malaria in children under five and in pregnant women. Ultimately, this would assist policy makers, planners and implements to improve on malaria policy, strategies and approaches for up-scaling level of information and uptake of preventive measures on malaria for achievement of national set targets [5].

\section{Methods ANd Materials}

Kisumu East Sub-County (KESC) was the area of study, which covers an area of 557.9 square kilometres. The area boarders Nyando Sub-County to the East, Nandi East to the North East, Emuhaya on the North, Kisumu West to the West and Rachuonyo on South across Lake Victoria. The population of the area was estimated at 392,321, with women of reproductive age at 102,004 and under five children at 62,771 in 2017 by using annual increase rate of 2.7\% [18].

The long rain is in months of March and May with short rains in September and November. The mean annual rainfall is $1280 \mathrm{~mm}$ with low altitude areas having 1000-1800 mm on average. Similarly, the area has a mean temperature range of $20^{\circ} \mathrm{C}$ to $30^{\circ} \mathrm{C}$ annually [19]. The Sub-County has high rates of malaria transmission and infection due to the climatic conditions attributed to the altitude, rainfall patterns, humidity and temperature that favour malaria's insect vector growth $[19,5,3]$.

This was a cross sectional survey that involved mothers of under-five children living in households within Kisumu East Sub-County, Kisumu County, Kenya. The study was conducted between September and October 2018.

Kisumu East Sub-County was purposively chosen due to its high numbers of malaria morbidity and mortality particularly among under five children and in pregnant women. The four locations of Konya West, Kolwa West, Migosi and Kolwa East were selected by random sampling technique giving locations equal chance of being enrolled in the study. Similarly, mothers with children were enrolled for this survey through snow balling sampling techniques with 96 of them each drawn from the four selected locations leading to 384 participants. The determination of sample size was by Fischer's formula for a target population of more than 10,000.

The representative sample was derived from the target population of women of reproductive age (15-49) years who were biological mothers of children under five living within the households in KESC, Kisumu County. For the purpose of this study, 384 mothers, public health officer (1), public health nurse (1) and location chiefs (4) participated in the research study. The sample size determination by Fisher formula.

Thus, $\boldsymbol{n}=\frac{z^{2} p q}{d^{2}}$

Where:

$\mathrm{n}=$ the desired sample size.

$\mathrm{Z}=$ the standard normal deviate at the required confidence level (1.96).

$\mathrm{p}=$ the proportion in target population estimated to have characteristics being measured (50\%).

$\mathrm{q}=1-\mathrm{p}(0.5)$

$\mathrm{d}=$ the level of statistical significance set at $(0.05)$.

$n=\frac{(1.96)^{2}(.50)(.50)}{(0.05)^{2}}=384$.

The researcher conducted transect-walk, followed by pre-testing of questionnaire on mothers and two interview guides on health providers and location chiefs. The questionnaire for mothers and interview guide for chiefs were piloted in Kolwa Central location. The interview guide for health provider was pre-tested in Kisumu West Sub-County in Kisumu County. The piloting of research instruments guaranteed their validity and reliability. Thereafter, questionnaires and interview guides were administered to mothers (main respondents), health providers and location chiefs (Key Informants) in order to collect information for this research study.

The data collected was edited, coded and organized into themes for completeness, consistency and accuracy. The KIs also gave information that were analysed through thematic procedures to support and clarify study findings. 
Data analysis was conducted by descriptive and inferential statistics while Chi-square tests were done considering a 0.05 significance level. Similarly, the SPSS for Windows Version 17 was used to statistically analysed data collected. The findings of the research study were presented in tables, figures, prose text and pictorials.

Approval for the study was given by the Catholic University of Eastern Africa (GABA) Department of Post Graduate Studies. A research permit was obtained from the National Commission for Science Technology and Innovation (NACOSTI) permit No. NACOSTI/P/18/13316/25111. The research protocols were observed that guaranteed privacy and confidentiality of participants.

\section{RESULTS}

\section{Demographic Characteristics}

Results on socio-demographic characteristics showed that, of $185(48 \%)$ mothers had attained Secondary level of education, $159(41 \%)$ had Primary and $37(10 \%)$ had College diploma or University degree. Similarly, 358 (93\%) of them joined self-help group and 5 (1\%) Cooperative Societies. Meanwhile, $352(92 \%)$ of mothers were married, 164 $(43 \%)$ were engaged in small-scale businesses, $153(40 \%)$ practiced subsistence farming while $66(17 \%)$ were employed.

\section{Level of Awareness about Malaria}

In total, 384 mothers participated in the study, up to $383(99 \%)$ of them were aware mosquitoes insect transmit malaria, $378(98 \%)$ of participants knew malaria is contracted by bites from mosquitos infected with malaria parasites (Table1).

Table-1: Mode of Malaria Transmission

\begin{tabular}{|l|l|l|}
\hline Responses & $(\mathrm{n})$ & $(\%)$ \\
\hline Drinking contaminated water & 6 & 2 \\
\hline Being rained on or exposed to cold weather & 0 & 0 \\
\hline Bite from mosquitoes infected with malaria parasites & 378 & 98 \\
\hline Others & 0 & 0 \\
\hline Total & $\mathbf{3 8 4}$ & $\mathbf{1 0 0}$ \\
\hline
\end{tabular}

Similarly, $300(78 \%)$ responders mentioned that, mosquitoes breed in stagnant waters or in swampy places while $83(22 \%)$ of them said they breed in bushes or in overgrown grass (Table 2).

Table-2: Mosquito Breeding Places

\begin{tabular}{|l|l|l|}
\hline Responses & $(\mathrm{n})$ & $(\%)$ \\
\hline In dark corners & 1 & 0 \\
\hline Stagnant waters or swamps & 300 & 78 \\
\hline In bushes or overgrown grass & 83 & 22 \\
\hline Total & $\mathbf{3 8 4}$ & $\mathbf{1 0 0}$ \\
\hline
\end{tabular}

Further findings established that, 361 (94\%) mothers were aware mosquitoes feed or bite at night while $23(6 \%)$ knew they feed or bite either during the day or at night. Results of hypothesis test established that, there was statistically significant association between level of malaria awareness and malaria prevention $(\mathrm{p}=0.023)$.

\section{Recognition of Signs and Symptoms of Malaria}

Multiple entry results found that, $380(100 \%)$ of mothers knew vomiting, $378(99 \%)$ recognized fever, 374 $(98 \%)$ identified loss of appetite while $102(27 \%)$ mentioned headache as signs and symptoms of malaria in children under-fives (Figure 1). 


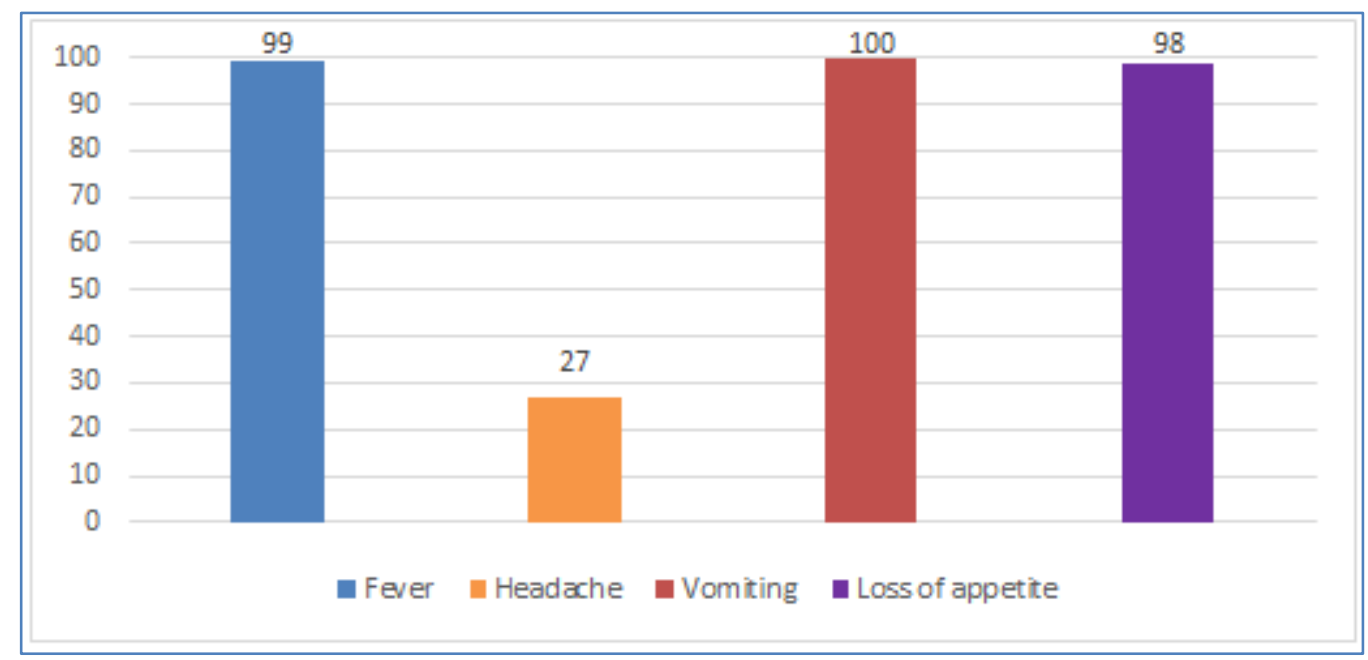

Figure 1: Signs and symptoms of malaria in children

Out of the total responders, $378(99 \%)$ recognized inability to eat, drink or beast feed, $378(99 \%)$ identified high body temperature or fever, $268(71 \%)$ mentioned convulsion while $245(64 \%)$ knew vomiting as severe signs and symptoms of malaria in under 5 children (Figure 2).

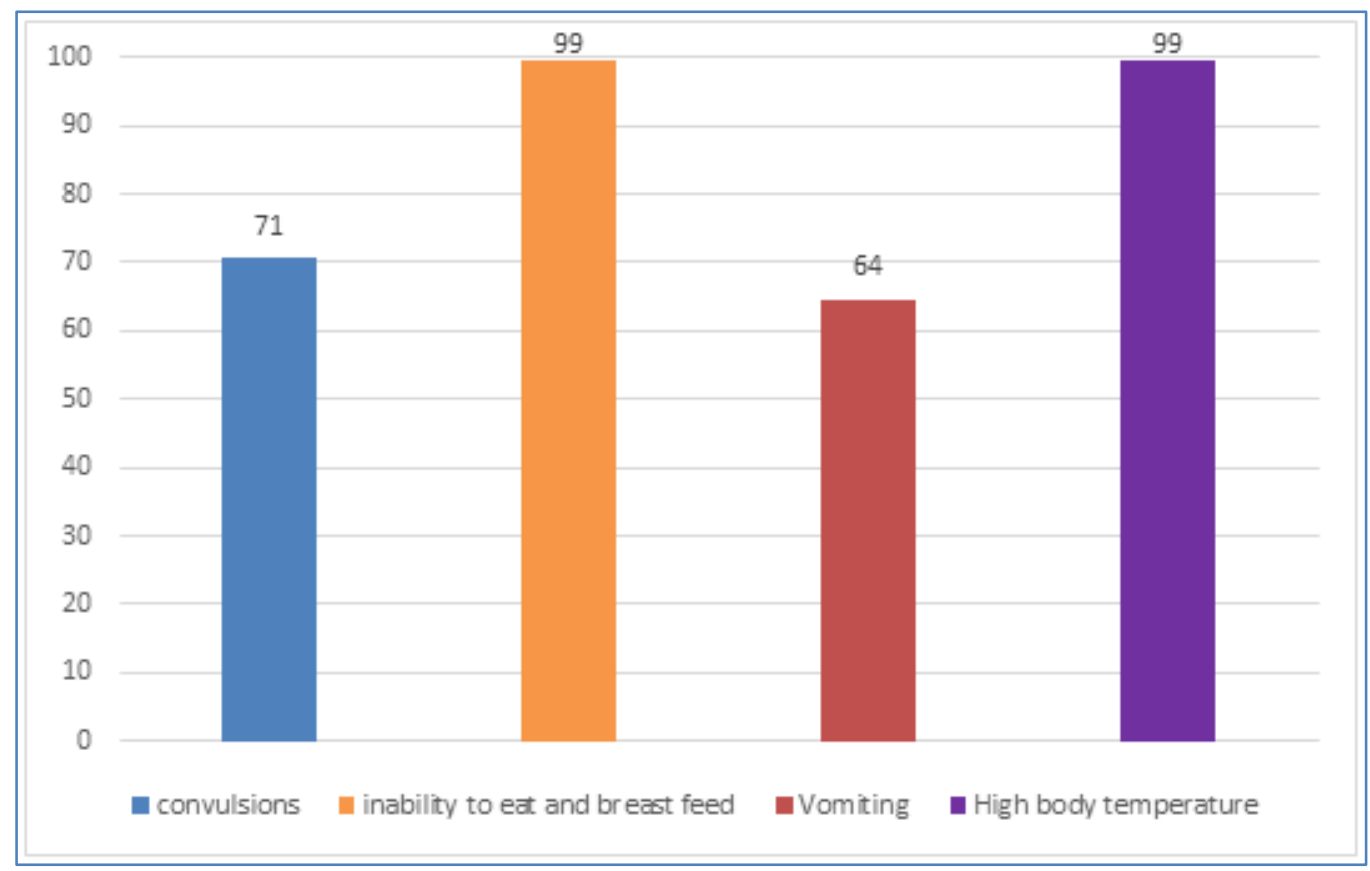

Figure 2: Signs and symptoms of severe malaria in children

Further data on multiple entries showed that, 357 (93\%) of mothers recognized headache, $339(88 \%)$ identified fever, $333(87 \%)$ mentioned loss of appetite and $283(74 \%)$ knew joint pain, backache and body weakness as common signs and symptoms of malaria in pregnancy (Figure 3). There was a statistically significant association between level of recognition of signs and symptoms of malaria and malaria prevention $(\mathrm{p}=0.039)$. 


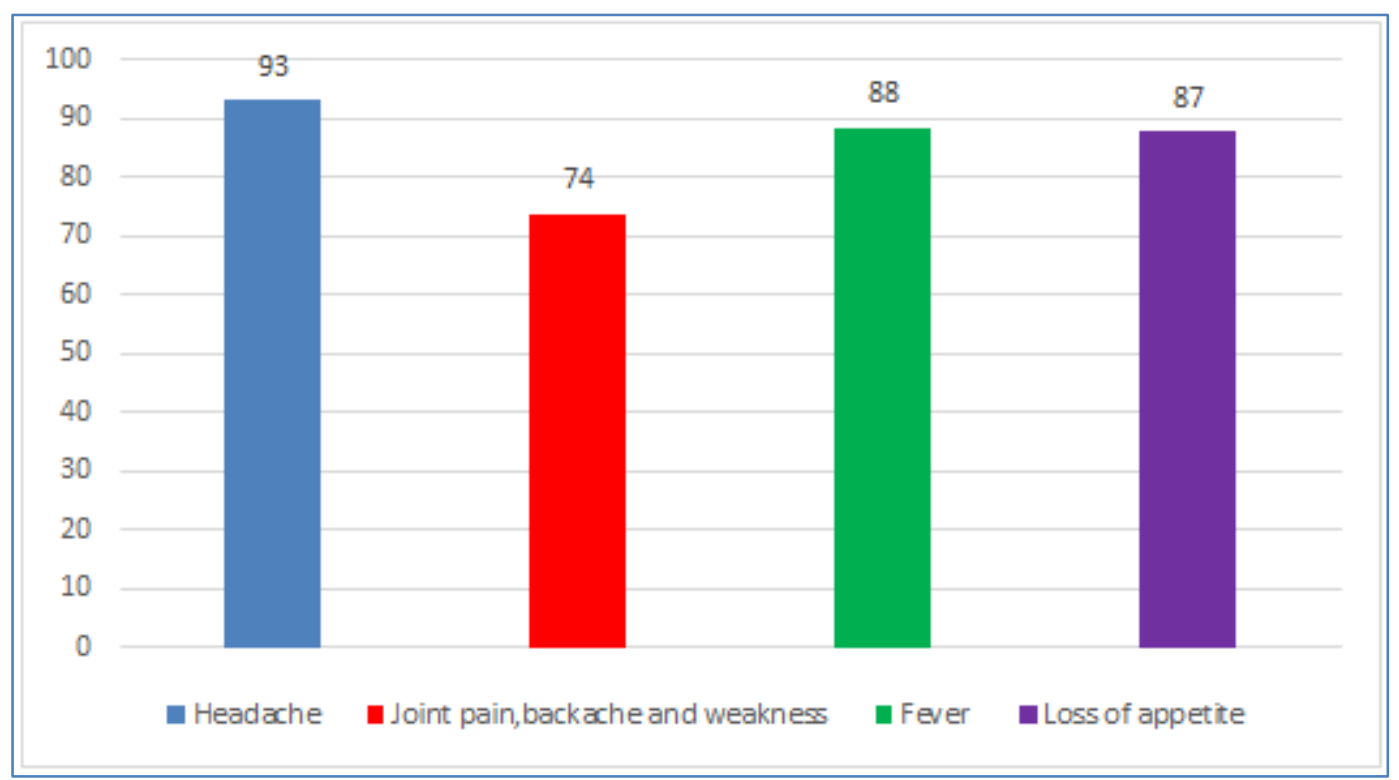

Fig-3: Common signs and symptoms of malaria in pregnancy

Health providers indicated that, there was a malaria program in Kisumu East Sub-County. The program main activities were distribution of mosquito nets, free treatment of children under-fives suffering from malaria, integrated antenatal care services, environmental management practices and health education. They stated that, mothers being primary care givers ensure their family utilize malaria preventive measures and pass on malaria information to the local population.

The chiefs reported that, malaria and HIV/AIDS were major health challenges in the area. However, despite awareness, inhabitants do not consider malaria as a serious health problem because "it is always amidst them". They further suggested that, vernacular Radio stations, television networks, public meetings and mobile telephony should be used more often to inform mothers on malaria related information for better health outcome.

\section{DISCUSSION}

\section{Awareness about Malaria}

From the findings, majority of mothers knew mosquitoes transmit malaria. Further data indicated that, most of them were also aware children under 5 contract malaria by bite of mosquitoes infected with malaria parasites. Similarly, some of them wrongly believed that the disease was transmitted by drinking contaminated waters. These findings resonated well with other similar studies, which indicated that many participants also knew malaria could be contracted through bite of mosquitoes having malaria parasites $[9,13]$.

Most mothers in this study, knew mosquitoes breed in stagnant waters or swampy places. However, despite high level of knowledge about their breeding sites, some of them still wrongly mentioned they breed in bushes or in overgrown grass. In contrast, a study in Nigeria reported that, almost half of all of interviewees did not know where mosquitoes breed [13]. Furthermore, low level of knowledge on breeding sites could have been occasioned by inadequate malaria campaign program, traditional beliefs or lack of information about the disease among population.

Further studies in Uganda and Nigeria had also reported misconceptions about malaria among participants. This could have been due to inadequate or lack of accurate information and misinformation or poor malaria campaign program in the areas [9, 13]. Misleading beliefs about malaria could undermine level of mothers' participation in malaria prevention program targeting under-fives and pregnant women in high risk regions similar to our study setting. These could lead to misdirecting efforts towards less effective interventions measures such as clearing of overgrown bushes and tall grass instead of draining stagnant waters or swampy places where mosquitoes breed.

Illustratively, lack of information and false beliefs about malaria could undermine use of mosquito nets or environmental management practices. This could also lead to large numbers of mosquitoes, bites and malaria cases among children and pregnant women leading to disabilities or deaths. Ultimately, the Sub-County would record many cases of malaria as reported by KIs particularly among vulnerable group disabilities, illnesses or deaths. 
Further findings established that many participants knew mosquitoes feed or bite at night while a very small proportion was aware they feed or bite during the day or at night. These findings concurred with those reported in a study in Uganda [9]. Our study further showed high levels of knowledge on malaria information among respondents. This could be attributed to a malaria campaign program rolled out in the region.

The area is a malaria endemic region where mosquitos' density, bites and cases of malaria are high among under 5 and in pregnant women [2, 5, 19]. This was confirmed by Key Informants when some of them stated that, malaria and HIV/AIDS were major health problems in KESC, Kisumu County, Kenya. Similar studies conducted in Uganda, Cameroon and Nigeria had posted almost similar results, despite diverse demographic, political and geographical characteristics $[9,11,12,13]$.

Overall data showed that there was high level of awareness about malaria related information among interviewees despite some misconceptions. Thus for sustainability, there should be concerted efforts to intensify and promote Advocacy, Communication and Social Mobilization (ACSM) program for behaviour change that would increase awareness level and help address misinformation about the disease. This could be achieved by use of the Radio, TVs network and Mobile telephony including Traditional Birth Attendants (TBAs) and Community Health Volunteers (CHVs) in local communities.

\section{Recognition of Signs and Symptoms of Malaria}

Majority of mothers knew signs and symptoms of malaria. They were also able to identify vomiting, loss of appetite, fever and headache as common signs and symptoms of malaria in children. The findings related to other studies which showed that many of the responders also knew of malaria and its signs and symptoms $[9,20,11,13,21]$. Further findings showed that, most mothers mentioned inability to eat, drink or breast-feed, high body temperature or fever, convulsion and vomiting as common signs and symptoms of severe malaria in under 5 children in the study area.

Similarly, many responders knew headache, fever, loss of appetite, joint pain, and backache and body weaknesses as common signs and symptoms of malaria in pregnancy. In comparison, a study in Nigeria also found that a small number of participants were not aware of malaria symptoms [13]. However, a study in Cameroon reported that, majority of respondents could only identify at least one sign or symptom of malaria [11]. Furthermore, other studies suggested fever as the most common sign of malaria $[9,11,3,20]$. Therefore, recognizing signs of fever in children within 24 hours is crucial for prompt health care services which would help to prevent malaria related complications in the group $[9,22,23,6]$.

Furthermore, current findings showed that, a majority of mothers recognized signs and symptoms of malaria in children under 5 . This could be because malaria is a leading cause of mortality and morbidity in children; it might also be as a result of KESC being in malaria endemic region and due to intensive malaria campaign program in the area. However, failure to identify signs and symptoms of malaria could undermine case management practices in under-fives. Consequently, this could lead to malaria related complications, as depicted in previous studies [22, 6, 3]. Thus, acquisition of information on malaria would enable mothers to respond promptly to malaria cases amongst children and in pregnant women in the area.

Therefore, there should be health education on how to recognize signs and symptoms of malaria in children under five and women. Similarly, lack of information about malaria could jeopardize mothers' participation in malaria prevention program that target under 5 children in high risk areas. It could also undermine case management protocols that lead to poor health outcome for most at risk group in the study area. For sustainability, both the County and National governments should implement ACSM malaria program in Kisumu East Sub-County, Kenya that enhance use of malaria preventive measures.

Previous studies had shown that, through use of Vernacular Radio stations, Televisions and mobile telephony the level of awareness on malaria could be up scaled [9,11,24,3,16,11]. Furthermore, previous studies showed that education could also improve level of awareness about malaria in a population $[20,11,13,8]$. These findings were supported by some of our key informants views suggesting the need for capacity building and empowerment for women to enable them participate in malaria prevention program in Kisumu East Sub-County.

In overall, our study determined that, a majority of mothers were able to recognize signs and symptoms of malaria in children under 5 years and among pregnant women. Thus, this could have enabled them to seek early health care intervention for their children within 24 hours of fever onset in health institutions and use of malaria preventive measures in the Sub-County. 


\section{CONCLUSION AND RECOMMENDATIONS}

Therefore, for sustainability, more efforts should be directed towards increasing level of information about malaria among mothers in the area. This would help to support behaviour change that enables them to participate effectively in malaria program at local level. It would also assist women identify signs and symptoms of malaria early enough in their children for health care intervention services within 24 hours. Moreover, stakeholders should direct their effort towards ACSM program for positive behaviour change in malaria program in the study area. These measures would eventually help in reducing malaria cases in children and among pregnant women for overall better health outcome in Kisumu East Sub-County, Kenya.

\section{Competing interests}

The authors declare that they have no competing interests.

\section{Authors' contributions}

Walter Ochieng Okande conceived, designed, collected data, analysed and wrote manuscript. Gilbert Bor and Fredrick Odoyo supervised and coordinated the design of the study.

\section{ACKNOWLEDGEMENTS}

The authors appreciate all those who participated in one way or another in this research study. We are most grateful to the village elders for accompanying the researcher during data collection process. This was part of a thesis for Master of Arts degree in Development Studies at the Catholic University of Eastern Africa by Walter Ochieng Okande. Similarly, we are most grateful for the ethical committee of NACOSTI for authorizing this study.

\section{FUNDING}

Walter Ochieng Okande was not provided with any financial support from any organization or agency for this research study.

\section{REFERENCES}

1. World Health Organization. (2016). World Malaria Report 2016. Global Malaria Programme. Geneva, Switzerland: World Health Organization.

2. Government of Kenya (MOH). (2016). The Epidemiology and Control Profile of Malaria in Kenya. Nairobi, Kenya: NMCP, MOH, Nairobi, Kenya.

3. Government of Kenya (MOH). (2015). Malaria Indicator Survey. Ministry of Health, Kenya National Bureau of Statistics.

4. Government of Kenya. (2018). Kenya Economic Survey (KES), 2018. Nairobi, Kenya: Kenya National Bureau of Statistics (KNBS).

5. Government of Kenya (MOH). (2009). National Malaria Strategy (NMS, 2009-2018). Nairobi, Kenya: Government Printers.

6. World Health Organization (WHO). (2004). Malaria in Pregnancy: Strategic Framework for Malaria Prevention and Control during Pregnancy in the African Region. Geneva, Switzerland: WHO.

7. Government of Kenya. (2007). Kenya Vision 2030. Nairobi, Kenya: Government Printers.

8. Adebayo, A. M., Akinyemi, O. O., \& Cadmus, E. O. (2015). Knowledge of malaria prevention among pregnant women \& female caregivers of under-five children in rural South West Nigeria. Peer J., 3, e792. DOI $10.7717 /$ peerj.792

9. Mwanje, F. L. (2013). Knowledge, Attitudes and Practices on Malaria Prevention and Control in Uganda: A case Study of Nsaabwa Village, Mukono District, Uganda. MAKSPH-CDC Fellow.

10. Musoke, D., Karani, G., Ssempebwa, J. C., Etajak, S., Guwatudde, D., \& Musoke, M. P. (2015). Knowledge and Practices on Malaria Prevention in two Rural Communities, Wakiso District in Uganda. African Health Sciences, $15(2), 401-412$.

11. Kimbi, K. H., Nkesa, B. S., Ndamukong-Nyanga, L. J., Sumbele, U. N. I., \& Atashili, J. (2014). Knowledge \& Perception towards Malaria Prevention among Vulnerable Groups in the Buea Health District, Cameroon. BMC Public Health, 14, Article number 883. http://www.biomedcentral.com/1471-2458/14/883

12. Akaba, G. O., Otubu, J. A. M., Agida, E. T., \& Onafowokan, O. (2018). Knowledge and utilization of malaria preventive measures among pregnant women at a tertiary hospital in Nigeria's federal capital territory. Nigerian Journal of Clinical Practice, 16(2), 201-206.

13. Oladimeji, K. E., Tsoko-Gwegweni, J. M., Ojewole, E., \& Younga, S. T. (2019). Knowledge of Malaria Prevention among Pregnant and Non-Pregnant Mothers of Children aged under 5 years in Ibadan, South West Nigeria. Malaria Journal, Global Health Research Policy. https://doi.org/10.1186/s12936-019-

14. Government of Kenya (MOH). (2015). Gender and Malaria in Kenya. Malaria Control Unit, Nairobi, Kenya. 
15. Government of Kenya (MoH). (2015). Gender and malaria in Kenya. Malaria Control Unit, Nairobi, Kenya.

16. Yaya, S., Uthman, O. A., Amouzous, A., \& Biswajit, G. (2018). Media Exposure \& its Impact on Malaria Prevention Behaviour among Adult Women in Sub Saharan Africa. Malaria Journal. Global Health Research Policy. https://doi.org/10.1186/s41256-018-0075-2

17. Gunn, J. K. L., Ernst, K. C., Centre, K. E., Biscotti, K., Nunez, A. V., Huynh, M., Okello, A., \& Hayden, M. H. (2018). Current Strategies and Successes in Engaging Women in Vector Control: A systematic review. Retrieved September 4.2019 from http://gh.bmj.com

18. Government of Kenya (KNBS). (2010). Kenya National Population and Housing Census (2009). Nairobi, Kenya: Government Printers.

19. County Government of Kisumu (CGK). (2018). Kisumu County Integrated Development Plan (2018-2022). The Department of Health and Sanitation, Kisumu, Kenya.

20. Feroro, D. A., Chaparro, P. E., Vallejo, A. F., Benavides, Y., Gutierrez, J. B., Arevalo-Herrera, M., \& Herrera, S. (2014). Knowledge, Attitude\& Practices of Malaria in Colombia. Malaria Journal, $13,165$. https://doi.org//10.1186/1475-2875-13-165

21. Government of Kenya. (2016). Kenya National Council for Population and Development. Nairobi, Kenya: KNBS.

22. Government of Kenya (MOH). (2012). Malaria Prevention and Treatment: A community Education Training Manual. Nairobi, Kenya: Ministry of Public Health and Sanitation.

23. Shulman, C. E., Dorman, E., \& Bulmer, J. N. (2002). Malaria as A cause of Severe Anaemia in Pregnancy. Lancet, 360-497. https://www.thelancet.com/action/showPdf?pii=S0140-6736\%2802\%2909662-9

24. Government of Kenya. (2016). National Malaria Communication Strategy of (NMCS, 2016-2021) (rev. ed.). Nairobi, Kenya: Government Printers. 